\title{
External Perforating Push Out Lateral Osteotomies for Treatment of Crooked Nose
}

\author{
Sameh Mahmoud Amin, MD, Tamer Omar Fawzy,MD, Wassim Mikhaiel, MD, Mohamed \\ Hosny, MSc.
}

Otorhinolaryngology department, Fayoum University, Fayoum, Egypt.

\begin{abstract}
Aim of the study: The Aim of the Study is to assess the efficacy of new technique of external perforating push out lateral osteotomies for correction of crooked nose.
\end{abstract}

Patients and Methods: The study was held on 20 patients (18 men and 2 women) with crooked noses who had both functional and cosmetic nasal deformities; with age group between 19 and 37 years. All cases were treated surgically by rhinoplasty using classic nasal osteotomies in addition to a newly modified external perforating push out lateral osteotomy. Patients were prospectively followed up for 6 months. Pre and post-operative assessment was done through standard photographs and computer programming for symmetry of both sides of the face, angel of deviated crooked nose and size of the depressed area.

Results: The symmetry of both sides of the face across the mid facial line at inter canthal and inter alar distance was slightly improved from $84.07 \%$ to 92.28 $\%$ and from $95.53 \%$ to $97.45 \%$ respectively. The most significant improvement of symmetry of both sides of face was achieved at the point of maximum deviation from $25.115 \%$ to $95.86 \%$. The mean angle of deviation changed from $138{ }^{\circ}$ Pre-operative to 180 ${ }^{\circ}$ Post-operative. Depressed area ranged pre-operative from $97.6 \mathrm{~mm} 2$ to $191.2 \mathrm{~mm} 2$ and was abolished completely post-operatively.

Conclusion: The External Perforating Push Out Lateral osteotomy is an effective technique for lateral repositioning of the bony lateral sidewall of the nose. It is an easy, accurate approach and provide great preservation of the periosteal support of the bony segments. This technique provides a more predictable long-term result, with preservation and improvement of the nasal airway.

Keywords: Push out, Osteotomies, Perforating, Crooked, Rhinoplasty

\section{Introduction}

Crooked nose has always been a surgical challenge for surgeons. It is of essential importance to achieve both functional and esthetic improvements.[1] Various techniques have evolved through times to attain correction of the deviated nose. Osteotomies are performed to correct deformities of the nasal bones during rhinoplasty. They can be used to: (1) straighten a deviation within the bony nasal vault; (2) close the open roof following dorsal hump reduction; and/or (3) narrow the bony nasal dorsum or sidewalls.[1] The four common osteotomies, in order of clinical use, are lateral, medial, intermediate, and crossroot.[1], [2]

Correction of Bony Pyramid can be achieved by Unilateral Lateral Osteotomy (Internal or External), Bilateral Lateral Osteotomies (in fracture in one side, out fracture in another side) and on lay graft Camouflage.

Selecting the appropriate technique will depend on specific aspects of the patient's anatomy, type of deformity, desired outcome and the cosmetic and functional affects the changes will have on the nose.[3] 
Routine nasal osteotomies will treat various deformities of the bony nasal vault effectively. Regarding treatment of crooked nose, additional types or modification to general osteotomies will be applied to ensure aesthetic and functional result.

The perforating intranasal osteotomy can be used to "pushout" the nasal bones that have been medially displaced by previous trauma or. The objective of the push-out technique is to move the nasal sidewall laterally in cases that are too narrow. The perforating osteotomy can be effective in difficult cases, such as revision rhinoplasty or posttraumatic nasal surgery, in which maintenance of structural support is critical.[1]

\section{Patients and Methods}

The study is prospective; it includes 20 patients. The patients were admitted from the outpatient clinic of Fayoum university hospitals, Fayoum city, Egypt, after being diagnosed with crooked nose. All underwent Rhinoplasty using an open approach in the period from June 2017 till December 2018. The patients were orally and officially consented using the standard form of the informed written consent. The study was approved by the medical ethics committee of the Faculty of Medicine, Fayoum University.

The patients had to fulfill the following inclusion criteria: they had to have post traumatic crooked nose and their age ranged from sixteen to fifty-six years. The exclusion criteria were the extremes of age,
General chronic diseases: i.e. cardiac, hepatic and renal diseases, and Nasal chronic diseases: i.e. Atrophic Rhinitis, rhinoscleroma and allergic rhinitis.

A full history should be obtained, including previous trauma, previous nasal surgery, nasal obstruction, any chronic disease either of nasal or general diseases as well as use of illicit drugs and tobacco. Frank discussion will be conducted regarding the patient perception of his or her nose and personal goals for rhinoplasty. Unrealistic expectations for surgery should be identified and noted.

Physical examination of the nose including palpations that informs about skin quality, tip support, alar cartilages, nasal bone and nasal septum, anterior rhinoscopy and endoscopic examination if needed.

Laboratory (Routine) investigations were done for all patients including complete blood count (CBC), ALT, AST, Urea, Creatinine, serum albumin, P.T and blood sugar

Radiological investigations such computerized tomography, ECG, and plain chest radiography in case of previous history of smoking, bronchial asthma, or clinical signs of chest troubles. 
Evaluation of the patients was assessed

subjectively and objectively. The

subjective assessment included

Rhinoplasty Outcome Evaluation (ROE)

questionnaire and standard photography.

The objective assessment of symmetry of

both sides of the face and angle of

deviation of crooked nose was conducted through Microsoft ${ }^{\circledR}$ Office Power point, while the size of the depressed area was measured by Imagej Computer Program. The push out technique was performed through an external approach, External Perforating push out lateral osteotomies done by: First tap diagonal to fix the osteotomy, second tap transverse with index finger protecting the eye and finally osteotomy performed to elevate the depressed nasal bone.

\section{Results}

This study includes twenty patients (18 men and 2 women) with crooked nose underwent Rhinoplasty. The study group of patients show predominance of males (18) with $90 \%$ in comparison to females (2) with only $10 \%$ within mean of age 25 +-5.7 .

Subjective assessment of group sample of patients was conducted through Rhinoplasty Outcome Evaluation (ROE) questioner and showed approximately 95 $\%$ satisfaction post-operatively compared with pre-operative level of satisfaction which was $10 \%$ with significant P-value $(<0.0001)$.

Standardized digital photographs for rhinoplasty were taken pre and post operatively; Then The photographs were rated as symmetrical or not by 3 independent observers for a global "first impression" of facial symmetry and the results showed facial asymmetry of $90 \%$ of the sample compared to post-operative results which showed restoration of facial symmetry in $95 \%$ of patients with significant $\mathrm{P}$-value $(<0.0001)$. 


\begin{tabular}{|l|c|c|}
\hline & Mean & SD \\
\hline \multicolumn{2}{|l|}{ Inter canthal/Inter alar distance } \\
\hline Pre-operative & $76.065 \%$ & 0.1 \\
\hline Post-operative & $76.175 \%$ & 0.1 \\
\hline P-value & $\mathbf{0 . 8 1 2}$ (NS) \\
\hline
\end{tabular}

Table 1: Comparison between group of the patients pre and post-operative of inter canthal /Inter alar distance.

Proportion between inter canthal to inter alar distance ranged from $60.7 \%$ to $95.3 \%$ preoperative \& ranged post-operative from $61 \%$ to $91.3 \%$ with overall change from 76.065 $\%$ pre-operative to $76.175 \%$ post-operative with no significant change in $\mathrm{P}$ value $(0.812)$ (Table 1).

\begin{tabular}{|c|c|c|}
\hline & Mean & SD \\
\hline \multicolumn{3}{|c|}{ Symmetry along Inter canthal ditance } \\
\hline Pre & $\Lambda \varepsilon . V$ & $V .9 V$ \\
\hline Post & 9Y.YA & $V . \varepsilon 0$ \\
\hline P-value & \multicolumn{2}{|c|}{$\mathbf{0 .} \cdots(\mathbf{S})$} \\
\hline
\end{tabular}

Table 2: Comparison between pre and post-operative Symmetry along inter canthal line.

Symmetry along Inter canthal ditance ranged from $72.7 \%$ to $100 \%$ pre-operative $\&$ ranged post-operative from $74 \%$ to $100 \%$ with overall change from $84.07 \%$ preoperative to $92.28 \%$ post-operative with significant $\mathrm{P}$ value $(0.001)$ (Table 2 ).

\begin{tabular}{|l|c|c|}
\hline & Mean & SD \\
\hline Symmetry along mid vault \\
\hline Pre-operative & ro.r & $9 .{ }^{\prime}$ \\
\hline Post-operative & 95.86 & $\varepsilon .91$ \\
\hline P-value & \multicolumn{2}{|c|}{$<\mathbf{0 . 0 0 0 1}$ (S) } \\
\hline
\end{tabular}

Table 3: Comparison between pre and post-operative of Symmetry along mid vault. 
Symmetry along mid vault line ranged from $12.7 \%$ to $47.6 \%$ pre-operative and ranged from $86 \%$ to $100 \%$ post-operative with overall range in this study from $25.115 \%$ to $95.86 \%$ with significant change in $\mathrm{P}$ value $(<0.0001)$ (Table 3$)$.

\begin{tabular}{|l|c|c|}
\hline & Mean & SD \\
\hline Symmetry along inter alar line \\
\hline Pre-operative & 90.54 & 5.29 \\
\hline Post-operative & 97.40 & 3.49 \\
\hline P-value & \multicolumn{3}{|c|}{$\mathbf{0 . 1 5 9}$ (NS) } \\
\hline
\end{tabular}

Table 4: Comparison between pre and post-operative Symmetry along inter alar distance.

Symmetry along inter alar distance (Figure 56) ranged from $\wedge \leqslant . r \%$ to $100 \%$ preoperative and ranged from $91.2 \%$ to $100 \%$ post-operative with overall range in this study from $95.54 \%$ to $97.4 \%$ with no significant change in P value (0.159) (Table 4)

\begin{tabular}{|c|c|c|}
\hline & Mean & SD \\
\hline \multicolumn{3}{|c|}{ Angel of deviation } \\
\hline Pre-operative & IrA. YO & 1.17 \\
\hline Post-operative & $1 \wedge \cdot . \cdot$ & $\because \cdot$ \\
\hline P-value & \multicolumn{2}{|c|}{$<0.0001(\mathrm{~S})$} \\
\hline
\end{tabular}

Table 5: Comparison between pre and post-operative Angel of deviation of crooked nose. Angle of deviation ranged from $122^{\circ}$ to $154^{\circ}$ pre-operative and changed from $138^{\circ}$ (Pre-operative Mean) to $180^{\circ}$ Post-operative with significant P-value $<0.0001$ (S) (Table $5)$.

\begin{tabular}{|l|c|c|}
\hline & Mean & SD \\
\hline Depressed Area (1) & 136.29 & 29.88 \\
\hline Depressed Area (2) & 137.38 & 29.43 \\
\hline P-value & \multicolumn{2}{|c|}{$<.0001$ (S) } \\
\hline
\end{tabular}

Table 6: Comparison between measurement of depressed area by two different subjects (1 and 2). 
Depressed area (Figure 58) ranged pre-operative by subject number 1 from $97.6 \mathrm{~mm} 2$ to $191.2 \mathrm{~mm} 2$ with average $136.24 \mathrm{~mm} 2$ while depressed area by subject number 2 ranged from $98.2 \mathrm{~mm} 2$ to $188.4 \mathrm{~mm} 2$. and both were completely abolished $(0 \mathrm{~mm} 2)$ postoperative. With significant P-value $(<0.0001)$ (Table 6).

Pre and post-operative comparison between subjective and objective assessment of symmetry of face, with no significant difference them at the three outcome parameters in our study respectively.

\section{Discussion}

The Crooked nose deformity is one of the most challenging corrections by rhinoplasty. In post-traumatic crooked nose there is a long or steep depressed side while the other side is short or shallow. Correction of the deviated nose especially the upper one third depends mainly on correction of deviated bony pyramid mostly done by osteotomies.[4]

In this study we tried to assess our patients subjectively and objectively. Patients' selfsatisfaction was subjectively assessed by the validated Rhinoplasty Outcomes Evaluation (ROE) questionnaire which originally developed by Alsarraf et al[5] and showed approximately $95 \%$ of patients believed that they had an excellent postoperative result in comparison with Lisandra et al [6] who had used ROE to assess the satisfaction of patients submitted to rhinoplasty to correct a crooked nose and assumed that approximately $90 \%$ of the patients in his study had a good or excellent postoperative result.

Subjective assessments for symmetry of both sides of the face was done by three independent observers for a global "first impression" of facial symmetry throughout standard photographs of patients. The results showed facial asymmetry of $90 \%$ of the pre-operative sample compared to post-operative results which showed restoration of facial symmetry in $95 \%$ of patients.

There was no significant difference in our study between subjective and objective assessments pre and post operatively compared to Paul Chatrath et al [7] which showed objectively, $97 \%$ of patients had significant degrees of facial asymmetry, with the midline to ala distances showing the most variations and the midline to oral commissures showing the least variations. Subjectively, $38 \%$ of results were perceived as asymmetrical, with the degree of the midline to the lateral alar margin asymmetry being an independent predictor of the perception of facial asymmetry on the binary logistic regression.

Objective assessment of the outcome parameters was mandatory to give a more realistic point of view of the surgical outcomes. 
It was conducted through standard photography and computer programing to measure several outcome parameters such as: symmetry of both sides of the face, the angle of deviation of the crooked nose and the size of depressed area.

Facial Asymmetry in Rhinoplasty Patients was assessed by Paul Chatrath et al [7] using (Adobe Photoshop Version 7.0; Adobe Inc, SanJose, Calif). And showed that Objectively, $97 \%$ of patients had significant degrees of facial asymmetry, with the midline to ala distances showing the most variations and the midline to oral commissures showing the least variations.

Symmetry of both sides of the face in our study was obtained in ratios" which were always calculated by dividing the smaller side by the larger one" in three different points (Inter Canthal, mid vault and inter alar) using Microsoft ${ }^{\circledR}$ Office Power point ${ }^{\circledR}$. Results showed that the slight increase of proportion of inter canthal distance to inter alar distance reflects the broadening of bony pyramid, despite the difference between pre and post-operative values is non-significant, but it reflects also the effect of using the external perforating push out osteotomy on lateralization of the depressed side of the bony pyramid.

The symmetry of both sides of the face across the mid facial line at inter canthal and inter alar distance was slightly improved from $84.07 \%$ to $92.28 \%$ and from $95.53 \%$ to $97.45 \%$ respectively; which reflects the overall improvement of symmetry of both sides of the face.

The most significant improvement of symmetry of both sides of face was achieved at the point of maximum deviation (at the line of mid vault which entails proportion of long segment of deviated nasal bone to short side) from $25.115 \%$ to $95.86 \%$ which mirrors the effect of bilateral in, out fracture osteotomies with external perforating push out lateral osteotomy on the depressed area.

Erdogan Okur et al [8] used the Scion Image computer program to measure angle of deviation in crooked nose pre and post operatively (both types $\mathrm{I}$ and $\mathrm{C}$ deviation) and found that $66.7 \%$ of all patients with crooked noses had good or excellent results after surgery according to success category ratings $(53.8 \%$ for $\mathrm{C}$ type and $78.6 \%$ for I-type crooked noses).

Yong-Ha Kim et al [9] used an Adobe software program (Adobe Photoshop, 7.0, Adobe system, San Jose, Calif) to measure the degree of nasal deviation objectively; Preoperative and postoperative anthropometric measurements revealed that the mean degree of deviation changed from $10.19^{\circ}$ to $3.43^{\circ}$, which was statistically significant.

Speaking of measurement of angle of deviation of crooked nose in our study was assessed by Microsoft ${ }^{\circledR}$ Office Power point ${ }^{\circ}$; Angle of deviation ranged from 
$122^{\circ}$ to $154^{\circ}$ pre-operative and the mean degree of deviation changed from $138^{\circ}$ (Pre-operative) to $180^{\circ}$ Post-operative.

In this study we tried to imply a new method for measuring a more sophisticated parameter which is the size of the depressed area in crooked nose using Image J Computer Program "with calibration of inter pupillary distance of each patient individually" on standard photographs with two independent rhinoplasty surgeons, there was an excellent reliability between depressed area measurements of the two subjects. Depressed area ranged pre-operative by the first subject from $97.6 \mathrm{~mm}^{2}$ to 191.2 $\mathrm{mm}^{2}$ with average $136.24 \mathrm{~mm}^{2}$ while depressed area by the second one ranged from $98.2 \mathrm{~mm}^{2}$ to $188.4 \mathrm{~mm}^{2}$ with average $137.38 \mathrm{~mm} 2$ and were abolished completely post-operatively.

External Perforating Push Out osteotomy has proved to be an easy approach to carry out and makes fracture with high precision along preset lines. The perforating technique has been noted to provide a better preservation of the periosteal attachments laterally, the bone stump is stable and there is much less damage to the nasal mucosa. There is reduced bleeding, edema and ecchymosis around eyes. The cutaneous scar at the entry site of the osteotomy is also invisible after the surgery.[10]

\section{Conclusions}

The External Perforating Push Out Lateral osteotomy is an effective technique for lateral repositioning of the bony lateral sidewall of the nose. It is an easy and accurate approach to perform and appears to provide great preservation of the periosteal support of the bony segments. This technique provides a more predictable long-term result, with preservation and improvement of the nasal airway. The procedure is ideal for managing the bony nasal segment that needs lateralization, particularly in posttraumatic crooked nose and revision rhinoplasty. Further studies are needed to assess the benefits of applying this modified new technique in the correction of the crooked nose. 


\section{References}

[1] S. P. Most and C. S. Murakami, "Nasal osteotomies: Anatomy, planning, and technique," Facial Plast. Surg. Clin., vol. 10, no. 3, pp. 279-285, 2002, doi: 10.1016/S1064-7406(02)00016-0.

[2] P. J. Byrne, W. E. Walsh, and P. A. Hilger, "The use of inside-out lateral osteotomies to improve outcome in rhinoplasty," Arch. Facial Plast. Surg., vol. 5, no. 3, pp. 251-255, 2003.

[3] R. J. Rohrich, W. P. Adams, J. Ahmad, and J. Gunter, Dallas rhinoplasty: nasal surgery by the masters. CRC Press, 2014.

[4] I. Koçak and E. Senturk, "Osteoplasty in crooked nose deformity: a novel approach," Aesthetic Plast. Surg., vol. 41, no. 3, pp. 628-636, 2017.

[5] R. Alsarraf, "Outcomes research in facial plastic surgery: a review and new directions," Aesthetic Plast. Surg., vol. 24, no. 3, pp. 192-197, 2000.

[6] L. M. Arima, L. C. Velasco, and R. S. L. Tiago, "Crooked nose: outcome evaluations in rhinoplasty," Braz. J. Otorhinolaryngol., vol. 77, no. 4, pp. 510-515, 2011.

[7] P. Chatrath, J. De Cordova, S. A. R. Nouraei, J. Ahmed, and H. A. Saleh, "Objective assessment of facial asymmetry in rhinoplasty patients," Arch. Facial Plast. Surg., vol. 9, no. 3, pp. 184-187, 2007.

[8] E. Okur, I. Yildirim, B. Aydogan, and M. A. Kilic, "Outcome of surgery for crooked nose: an objective method of evaluation," Aesthetic Plast. Surg., vol. 28, no. 4, pp. 203-207, 2004.

[9] Y.-H. Kim, C. Y. Jung, K. J. Chung, J. H. Lee, and T. G. Kim, "A systematized strategy in corrective rhinoplasty for the Asian deviated nose," Ann. Plast. Surg., vol. 79, no. 1, pp. 7-12, 2017.

[10] R. J. Rohrich, J. J. Minoli, W. P. Adams, and L. H. Hollier, "The lateral nasal osteotomy in rhinoplasty: an anatomic endoscopic comparison of the external versus the internal approach," Plast. Reconstr. Surg., vol. 99, no. 5, pp. 1309-1312, 1997. 